\title{
Best practices from nano-risk analysis relevant for other emerging technologies
}

Grieger, Khara Deanne; Jones, Jacob L.; Hansen, Steffen Foss; Hendren, Christine Ogilvie; Jensen, Keld Alstrup; Kuzma, Jennifer; Baun, Anders

Published in:

Nature Nanotechnology

Link to article, DOI:

$10.1038 / \mathrm{s} 41565-019-0572-1$

Publication date:

2019

Document Version

Peer reviewed version

Link back to DTU Orbit

Citation (APA):

Grieger, K. D., Jones, J. L., Hansen, S. F., Hendren, C. O., Jensen, K. A., Kuzma, J., \& Baun, A. (2019). Best practices from nano-risk analysis relevant for other emerging technologies. Nature Nanotechnology, 14(11), 9981001. https://doi.org/10.1038/s41565-019-0572-1

\section{General rights}

Copyright and moral rights for the publications made accessible in the public portal are retained by the authors and/or other copyright owners and it is a condition of accessing publications that users recognise and abide by the legal requirements associated with these rights.

- Users may download and print one copy of any publication from the public portal for the purpose of private study or research.

- You may not further distribute the material or use it for any profit-making activity or commercial gain

- You may freely distribute the URL identifying the publication in the public portal 
Grieger et al., 2019, Nature Nanotechnology

\title{
What are the Key Best Practices from Nanomaterial Risk Analysis That May Be Relevant for Other Emerging Technologies?
}

\author{
Khara Grieger ${ }^{1}$, Jacob L. Jones ${ }^{2}$, Steffen Foss Hansen ${ }^{3}$, Christine Ogilvie Hendren ${ }^{4-5}$, Keld Alstrup Jensen ${ }^{6}$, \\ Jennifer Kuzma ${ }^{1}$, Anders Baun ${ }^{3}$ \\ ${ }^{1}$ Genetic Engineering and Society Center, North Carolina State University, Raleigh, NC, USA; \\ ${ }^{2}$ Department of Materials Science and Engineering, North Carolina State University, Raleigh, NC, USA; \\ ${ }^{3}$ Department of Environmental Engineering, Technical University of Denmark, Kgs. Lyngby, Denmark; \\ ${ }^{4}$ Department of Civil and Environmental Engineering, Duke University, Durham, NC, USA; \\ ${ }^{5}$ Center for the Environmental Implications of NanoTechnology, Duke University, Durham, NC, USA \\ ${ }^{6}$ National Research Centre for the Working Environment, Copenhagen, Denmark
}

The experiences gained from the last 15 years of nanomaterial risk analysis may be useful for risk analysis efforts of other emerging technologies.

It has been 15 years since the first wave of international research programs were launched to understand the potential health, environmental, and societal impacts of nanotechnologies and engineered nanomaterials [1]. Among other areas, investigations into the adequacy of the chemical risk assessment framework for nanomaterials soon became a keystone research effort [2]. While great strides have been made [3], including key work by national and international research consortia (e.g., OECD Working Party for Manufactured Nanomaterials, EU's NanoSafety Cluster, US National Nanotechnology Initiative) [4-6], uncertainty still significantly impedes nanomaterial risk evaluations. Today concrete conclusions regarding the health and environmental risks of most nanomaterials and products in which they are contained are lacking, and regulations struggle to keep pace with the rapidly 
evolving science and data developments that underpin nanomaterial risk assessment $[6,7]$. Moreover, the reliance on risk assessment for informing oversight and decision-making for nanomaterials has not only led to numerous challenges $[6,8,9]$, due to the complexities and uncertainties involved in characterizing nanomaterials and associated risks, but has also illuminated several underlying and unresolved tensions regarding the use of specific, existing test methods and risk assessment frameworks for decision making [10].

Given the experiences, challenges, and lessons learned over the last 15 years, it is now an opportune time to reflect on the field of nanomaterial risk assessment and analysis and, perhaps even more importantly, identify best practices that may be applicable to other emerging and disruptive technologies (e.g., synthetic biology, quantum technologies, artificial intelligence and machine learning, 3-D printing, climate engineering ). We have identified five best practices from nanomaterial risk analysis that in our view could help advance risk analysis and responsible innovation relevant for other emerging technologies.

\section{Promote Research Tailored for Regulatory Decision-Making}

While the difficulty of drawing concrete conclusions from risk assessments of nanomaterials and nanoenabled products is partly due to technical and analytical challenges $[3,7]$, there are also more fundamental issues at play: nano-risk research has largely been directed towards understanding the science rather than meeting decision-making and regulatory needs $[6,11]$. In this context, a distinction may be made between "traditional science" (i.e., hypothesis, discovery-based science that is not necessarily relevant for regulation, but highly relevant in scientific terms) and "regulatory-relevant science" (i.e., science designed to support regulatory decision-making). Numerous authors have previously highlighted the challenge of supporting decision-making needs while also developing the scientific areas of nanomaterial risk analysis and nano-safety $[6,8,9]$. While it is possible to link evolving nano-safety data to decision and policy-relevant needs using "bottom-up" strategies (e.g. [7]), the 
Grieger et al., 2019, Nature Nanotechnology

initiation of strategic, purposeful regulatory-relevant science programs (i.e., using "top-down" strategies) at the start of major risk and safety efforts for emerging technologies could help target research more effectively towards regulatory decision-making, at least for near- and medium-term decisions. This would also help to address the so called "pacing problem" often experienced between new technological innovation, efforts to understand their potential impacts, and subsequent regulatory responses [12].

\section{Set Realistic Time, Cost, and Complexity Estimates to Develop Risk Analysis}

Further refinements of risk assessment strategies, including the development of robust testing procedures and datasets for diverse nanomaterials, will likely continue to be an evolving process. In addition, the diversity of nanomaterial types, sizes, coatings, functionalizations, and products in which they are used only exacerbates existing challenges and amplifies the time, resources, and complexities involved. Although it may be disappointing that after almost two decades of research and significant investments in nanomaterial risk analysis (i.e., estimated at several hundred million to a billion US dollars/euros $[13,14])$, additional time and resources are needed to comprehensively understand potential impacts of nanomaterials, this may not be entirely surprising upon reflection. For comparison, it took over three decades to develop robust chemical risk assessments [15], two decades to develop quantitative microbial risk assessments in food [16], and similar timelines in other risk assessment fields [17]. In fact, early estimates anticipated that it would cost $\$ 249$ million to $\$ 1.18$ billion and take $24-53$ years to develop and complete quantitative risk assessments for nanomaterials and products [18].

The process of identifying risks, adapting or developing assessment protocols and procedures, and testing, validating, and harmonizing risk assessment methods for other emerging technologies are also likely be complex, time-consuming, and expensive. This may especially be the case if this process is based on the traditional approach of relying on experimental evidence and knowledge-based assessments for risk evaluations [17]. Therefore, it may help prepare and align stakeholder expectations 
Grieger et al., 2019, Nature Nanotechnology

early on to have realistic estimates of the time, costs, and degrees of complexities involved to derive concrete conclusions regarding risks. These estimates may help prepare industry, policymakers, and other decision-makers so that they can prioritize research efforts and funding programs directed at near-term methods, policy or decision-making while the underlying safety science is developed.

Estimates also need to factor in the often iterative process between industry, regulators, and other stakeholders when submitting safety data and information on new materials or technologies to ensure the current state-of-science is reflected in submissions.

\section{Develop Strategies to Deal with Uncertainties in Risk Analysis and Decision-Making}

Scientific uncertainty - defined here as all types and forms of knowledge deficits in evaluations [19, 20] has been one of the main obstacles in nanomaterial risk analysis [3, 21]. In response, additional nanosafety research was called for and launched in accordance (e.g., $[2,22,23])$. While many knowledge gaps have been reduced, new research challenges and areas of uncertainty have also arisen [6]. To help overcome these challenges related to the prevalence of extensive uncertainties in nanomaterial data and risk analysis, various alternative approaches have been proposed (e.g., risk-screening, prioritization, control banding, decision-support tools) [24]. However, a core issue of how best to deal with uncertainties in diverse nano-risk evaluations has not yet been addressed head-on despite several calls $[11,21,25,26]$.

In general, standard approaches to handle uncertainties in risk assessment (e.g., probabilistic analysis) may not be well-suited for emerging technologies characterized by deep and extensive uncertainties in potential risk evaluations [17]. Risk assessment efforts for nanomaterials and other emerging technologies would benefit from including or being complemented by uncertainty assessments that identify and describe different scientific uncertainties and communicate how they may impact overall risk estimates [27]. Dynamic risk evaluation and management processes also allow for adaptive responses to quickly evolving scenarios or in light of new information [28]. Adaptive and responsible risk 
Grieger et al., 2019, Nature Nanotechnology

governance frameworks that account for uncertainty in risk evaluations [26], incorporate stakeholder perspectives, and include procedural robustness may also be useful to proactively deal with uncertainty in risk analysis and decision-support involving emerging technologies [29].

\section{Develop Mechanisms to Share Risk Data while Protecting Privacy, Confidentiality, and Proprietary Information}

Concrete conclusions regarding the potential risks of nanomaterials have also been hampered by challenges related to data management and harmonization, along with issues of privacy, confidentiality, and intellectual property. Integrating diverse data sets without harmonized data production, collection and management approaches has led to mismatched datasets [30] and varying levels of completeness, quality, transparent documentation, and use of discrete data [6, 31]. Data access and sharing across projects and consortia have also been an obstacle, although some projects have started to implement findable, accessible, interoperable and reusable (FAIR) principles in response $[6,7]$. There have also been challenges to obtaining confidential or intellectual property-comprised information from industry [31], with modest results obtained thus far [31, 32].

Moving forward, having more harmonized, multi-scale, and even decision-directed approaches may help avoid challenges related to data harmonization and integration. It is recognized, however, it may be difficult to develop harmonized data management, data access, and ontologies in a quickly evolving field across research consortia. Future risk assessment and management efforts could rely on robust communication mechanisms between researchers and, with appropriate funding, integrate risk research efforts with respect to curation functionality, infrastructure, and communication processes from the onset. To help foster data sharing from industry and other stakeholders, key initiatives should focus on establishing strong relationships to build trust and increase communication as well as providing clear incentives for data sharing [33]. 
Grieger et al., 2019, Nature Nanotechnology

\section{Critically Evaluate and Select Robust, Fit-For-Purpose Tools for Risk Analysis}

To understand and quantify potential risks of nanomaterials for use in regulatory contexts, the chemical risk assessment framework was selected as the starting point, as highlighted above. This decision was made amidst early calls for broader risk governance approaches [34] and without significant or extensive stakeholder dialogue specifically focused on identifying and selecting appropriate and fit-for-purpose evaluation approach(es). While the use of the chemical risk assessment framework for nanomaterials was a logical step, given its use for chemicals, stakeholders may have benefited from being involved in an initial process that critically evaluated a range of tools, frameworks, and methods to evaluate risks and make subsequent decisions. A thorough and critical evaluation of robust, fit-for-purpose risk analysis tools or frameworks could have improved stakeholders' understanding and expectations on their utility, limitations, and outcomes, as well as helped illuminate the expected time, cost, and degrees of complexity that may be expected to eventually complete assessments. For example, probabilistic risk analysis, microbial risk analysis, and pest risk analysis have been proposed for active nanomaterials that interact with and respond to biological systems [35]. Further, not all decisions regarding potential risks need to be made from quantitative estimates, as other options include the selection of alternatives [36, 37]. Finally, entirely new ways of thinking about risks for emerging technologies may also be warranted in some cases, such as the utilization of "risk innovation" that incorporates risk appraisals within evolving innovation processes [38].

While pursuing and deriving (quantitative) risk assessment for nanomaterials and other emerging technologies is clearly worthwhile in some cases (e.g., following "traditional science" processes), it may also be a lengthy and time-consuming endeavor, and there may be other risk evaluation approaches that could be more applicable for a given decision. A process that critically evaluates diverse evaluation frameworks and approaches followed by a transparent selection process for an emerging technology could be beneficial early-on to ensure that the most fit-for-purpose risk analysis framework is selected 
Grieger et al., 2019, Nature Nanotechnology

for utilization, further exploration, or to ultimately produce outcomes that meet decision-makers' needs. Convergence and team science methodologies may offer identification of useful approaches and methodologies and even derive new procedures to solving complex and emerging risks with greater societal relevance, including issues raised by nanomaterials and other emerging technologies.

\section{Acknowledgments}

The authors gratefully acknowledge support for this work through diverse funding mechanisms. In particular, KG and JLJ acknowledges the Game-Changing Research Incentive Program (GRIP) funded through the NC State Office of Research, Innovation, and Economic Development (ORIED), RTI International, and the Kenan Institute for Engineering, Science and Technology. KG and JK gratefully acknowledge the partial support of the Genetic Engineering and Society Center at NC State (go.ncsu.edu/ges). JLJ also acknowledges NSF award ECCS-1542015 and the Research Triangle Nanotechnology Network (RTNN), a site in the National Nanotechnology Coordinated Infrastructure (NNCI). AB and KAJ acknowledges the Horizon 2020 project, caLIBRAte for support (Grant agreement no. 686239). SFH wishes to acknowledge the Swedish Foundation for Strategic Environmental Research (Mistra) project Environmental Nanosafety Phase II. Finally, CO Hendren acknowledges support from the National Science Foundation (NSF) and the Environmental Protection Agency (EPA) under NSF Cooperative Agreement DBI-1266252 and EF-0830093, the Center for the Environmental Implications of NanoTechnology (CEINT). 


\section{References}

1. European Commission, Nanotechnologies: a Preliminary Risk Analysis on the Basis of a Workshop Organized in Brussels on 1-2 March 2004 by the Health and Consumer Protection Directorate General of the European Commission. 2004, European Commission.

2. Scientific Committee on Emerging and Newly-Identified Health Risks (SCENIHR), Opinion on the appropriateness of the risk assessment methodology in accordance with the technical guidance documents for new and existing substances for assessing the risks of nanomaterials. 2007, Scientific Committee on Emerging and Newly-Identified Health Risks (SCENIHR).

3. Oomen, A.G., et al., Risk assessment frameworks for nanomaterials: Scope, link to regulations, applicability, and outline for future directions in view of needed increase in efficiency. Nanolmpact, 2018. 9: p. 1-13.

4. $\mathrm{OECD}$, Investigating the different types of risk assessments of manufactured nanomaterials: Identifying tools available for risk management measures and uncertainties driving nano-specific data needs, in Series on the Safety of Manufactured Nanomaterials 2018, Organisation for Economic Co-operation and Development.

5. National Nanotechnology Initiative, National Nanotechnology Initiative Strategic Plan, in Washington, DC. 2016, Subcommittee on Nanoscale Science, Engineering, and Technology Committee on Technology of the National Science and Technology Council.

6. Prosafe, The ProSafe White Paper: Towards a more effective and efficient governance and regulation of nanomaterials. 2017. p. 1-46.

7. Jantunen, A.P.K., A. Mech, and K. Rasmussen, Workshop on Regulatory Preparedness for Innovation in Nanotechnology. 2018, Joint Research Centre (JRC), European Commission: Luxembourg.

8. Linkov, I., E. Anklam, Z.A. Collier, D. DiMase, and O. Renn, Risk-based standards: integrating topdown and bottom-up approaches. Environment Systems and Decisions, 2014. 34(1): p. 134-137.

9. Wickson, F., et al., Balancing scientific tensions. Nature Nanotechnology, 2014. 9(11): p. 870.

10. Miller, G. and F. Wickson, Risk analysis of nanomaterials: Exposing nanotechnology's naked emperor. Review of Policy Research, 2015. 32(4): p. 485-512.

11. Hjorth, R., The shortfall of risk assessment for decision-making. Nature nanotechnology, 2017. 12(12): p. 1109-1110.

12. Marchant, G.E., Addressing the Pacing Problem. 2011, Springer Netherlands: Dordrecht. p. 199205.

13. National Nanotechnology Initiative, National Nanotechnology Initiative Supplement to the President's 2019 Budget. 2018, Subcommittee on Nanoscale Science, Engineering, and Technology Committee on Technology of the National Science and Technology Council: Washington, DC.

14. NNI. Environmental, Health, and Safety Issues. 2019 [cited 2019 April 5]; Available from: https://www.nano.gov/you/environmental-health-safety.

15. Syberg, K. and S.F. Hansen, Environmental risk assessment of chemicals and nanomaterials The best foundation for regulatory decision-making? Science of the Total Environment, 2016. 541: p. 784-794.

16. Dennis, S., R. Buchanan, and A. Miller, Microbial Risk Assessment: Achievements and Future Challenges. Food Safety Magazine, 2002. December 2001/January 2002.

17. Aven, T., Risk assessment and risk management: Review of recent advances on their foundation. European Journal of Operational Research, 2016. 253(1): p. 1-13. 
18. Choi, J.Y., G. Ramachandran, and M. Kandlikar, The impact of toxicity testing costs on nanomaterial regulation. Environmental Science \& Technology, 2009. 43(9): p. 3030-3034.

19. EFSA, Guidance on Uncertainty in EFSA Scientific Assessment - DRAFT. 2015, European Food Safety Authority. p. 1-219.

20. Aven, T., et al., Risk Analysis: Fundamental principles, in Core Subjects of Risk Analysis. 2018, Society for Risk Analysis. p. 1-5.

21. Grieger, K.D., S.F. Hansen, and A. Baun, The known unknowns of nanomaterials: Describing and characterizing uncertainty within environmental, health and safety risks. Nanotoxicology, 2009. 3(3): p. 1-U17.

22. European Commission. Proceedings of the workshop on research projects on the safety of nanomaterials: reviewing the knowledge gaps. 2008. Brussels.

23. EPA, U.S., U.S. Environmental Protection Agency Nanotechnology White Paper, U.S. EPA, Editor. 2007, Nanotechnology Working Group, Science Policy Council, U.S. Environmental Protection Agency: Washington, DC.

24. Jantunen, A.P.K., S. Gottardo, K. Rasmussen, and H.P. Crutzen, An inventory of ready-to-use and publicly available tools for the safety assessment of nanomaterials. Nanolmpact, 2018.

25. Subramanian, V., et al., Sustainable nanotechnology decision support system: bridging risk management, sustainable innovation and risk governance. Journal of Nanoparticle Research, 2016. 18(4): p. 1-13.

26. Grieger, K.D., A. Baun, and R. Owen, Redefining risk research priorities for nanomaterials. Journal of Nanoparticle Research, 2010. 12(2): p. 383-392.

27. (EFSA), E.F.S.A., Guidance on Uncertainty Analysis in Scientific Assessments. EFSA Journal, 2017. 16(1): p. 5123.

28. Ramachandran, G., et al., Recommendations for oversight of nanobiotechnology: dynamic oversight for complex and convergent technology. Journal of Nanoparticle Research, 2011. 13(4): p. $1345-1371$.

29. Kuzma, J., Governance of genetically engineered animals in the wild: A need for procedurallyrobust risk analysis. Regulation and Governance, 2019.

30. Karcher, S., et al., Integration among databases and data sets to support productive nanotechnology: Challenges and recommendations. Nanolmpact, 2018. 9: p. 85-101.

31. Kuzma, J. and A. Kuzhabekova, Nanotechnology, voluntary oversight, and corporate social performance: does company size matter? Journal of Nanoparticle Research, 2011. 13(4): p. $1499-1512$.

32. Lai, R.W.S., et al., Regulation of engineered nanomaterials: current challenges, insights and future directions. Environmental Science and Pollution Research, 2018. 25(4): p. 3060-3077.

33. Jantunen, A.P.K., A. Mech, and K. Rasmussen, Workshop on Regulatory Preparedness for Innovation in Nanotechnology, in JRC Conference and Workshop Reports. 2018, European Union: Luxembourg.

34. Bowman, D.M. and G.A. Hodge, Nanotechnology and Public Interest Dialogue: Some International Observations. 2007. 27(2): p. 118-132.

35. Kuzma, J. and J.P. Roberts, Is adaptation or transformation needed? Active nanomaterials and risk analysis. Journal of Nanoparticle Research, 2016. 18(7): p. 1-18.

36. Linkov, I., et al., Comparative, collaborative, and integrative risk governance for emerging technologies. Environment Systems and Decisions, 2018. 38(2): p. 170-176.

37. Trump, B.D., D. Hristozov, T. Malloy, and I. Linkov, Risk associated with engineered nanomaterials: Different tools for different ways to govern. Nano Today, 2018.

38. Maynard, A.D., Why we need risk innovation. Nature Nanotechnology, 2015. 10: p. 730. 
Grieger et al., 2019, Nature Nanotechnology 